\title{
Diagnostic value of fine-needle aspiration combined with ultrasound for thyroid cancer
}

\author{
JUAN LI $^{*}$, QIAN WANG $^{2 *}$, LIANGLIANG WANG ${ }^{2}$, JING WANG $^{2}$, DONGXIAO WANG $^{1}$, \\ ZHAOQIN XIN ${ }^{2}$, YILIN LIU ${ }^{1}$ and QINGHUA ZHAO ${ }^{2}$ \\ Departments of ${ }^{1}$ Special Inspection and ${ }^{2}$ Ultrasound, Liaocheng People's Hospital, \\ Liaocheng, Shandong 252000, P.R. China
}

Received July 27, 2018; Accepted May 23, 2019

DOI: $10.3892 / \mathrm{ol} .2019 .10584$

\begin{abstract}
Diagnostic value of fine-needle aspiration (FNA) combined with ultrasound for thyroid cancer was evaluated. A retrospective analysis was performed on the preoperative FNA and ultrasound data of 165 thyroid nodule patients, were divided into group $\mathrm{A}(\leq 1 \mathrm{~cm}$ group) and group $\mathrm{B}$ ( $>1 \mathrm{~cm}$ group) based on the maximum diameter line of the thyroid nodule. The sensitivity, specificity, accuracy, positive predictive value and negative predictive value of FNA, ultrasound and FNA combined with ultrasound in the diagnosis of thyroid cancer were analyzed and compared. In group A ( $\leq 1 \mathrm{~cm}$ group), the sensitivity, specificity, accuracy, positive predictive value and negative predictive value of FNA in the diagnosis of thyroid cancer were $93.33,71.43,91.04,96.55$ and $55.56 \%$, respectively, those of ultrasound were 86.67, 28.57, 80.60, 91.23 and $20 \%$, respectively, and those of FNA combined with ultrasound were $100,28.57,92.54,92.6$ and $100 \%$, respectively, with statistically significant differences in the sensitivity, specificity, accuracy, positive predictive value and negative predictive value between FNA, ultrasound and FNA combined with ultrasound $(\mathrm{P}<0.05)$. In group $\mathrm{B}$, the sensitivity, specificity, accuracy, positive predictive value and negative predictive value of FNA in the diagnosis of thyroid cancer were 100, 54.55, 94.90, 94.57 and $100 \%$, respectively, those of ultrasound were 96.55 , $72.73,93.88,96.55$ and $72.73 \%$, respectively, and those of FNA combined with ultrasound were 100, 63.64, 97.96, 95.92 and $100 \%$, respectively, without statistically significant differences in the sensitivity, specificity, accuracy, positive predictive value and negative predictive value between FNA, ultrasound and FNA combined with ultrasound $(\mathrm{P}>0.05)$. FNA combined
\end{abstract}

Correspondence to: Dr Liangliang Wang, Department of Ultrasound, Liaocheng People's Hospital, 67 Dongchang West Road, Liaocheng, Shandong 252000, P.R. China

E-mail: wew9ez@163.com

*Contributed equally

Key words: fine-needle aspiration, ultrasound, combined detection, thyroid cancer, diagnostic value with ultrasound significantly improved the sensitivity and accuracy in the diagnosis of the thyroid nodule in group A, but it did not significantly improve the accuracy, sensitivity and specificity in the diagnosis of the thyroid nodule in group B.

\section{Introduction}

Thyroid cancer, a common malignancy of head and neck, has a high incidence of 56 cases per 100,000 persons that, ranks first in systemic endocrine tumors, and its incidence in females is higher than that in males $(1,2)$. In recent years, due to the deterioration of the environment and the accelerated pace of life, the incidence of thyroid cancer has increased posing a great threat to human life (3).

The tumor cells of thyroid cancer are derived from thyroid epithelial cells, and thyroid cancer has a high incidence and less obvious clinical symptoms in the early stage, so its early diagnosis is also increasingly important (4). At present, the thyroid nodule is often detected by FNA, isotope scanning and ultrasound in clinical practice (5). Ultrasound detection has the advantages of low price, non-invasiveness, simple operation and good resolution and commonly used in the examination of thyroid lesion clinically (6). It also provides detailed data and description on the size, location and morphology of the nodule during the examination of the thyroid nodule, and explores some clinically untouched nodules, which has a certain value in the early diagnosis of thyroid cancer (7). According to the research report of Niu et al (8), ultrasound can find the smallest thyroid nodule with a diameter of $1 \mathrm{~mm}$, and show the structure, envelope and calcification of the nodule with a diameter of $2 \mathrm{~mm}$ in detail. However, the polypathia of some nodules leads to the overlapping of ultrasound images, which causes ultrasound to have certain limitations and deficiencies for the diagnosis of benign and malignant small thyroid nodules. Because of its diagnostic safety, minimal invasiveness and good accuracy, FNA is currently considered to be the most effective method for the clinical diagnosis of thyroid malignancies in addition to pathological examination (9). At present, there are 6 classification criteria for FNA in the diagnosis of thyroid cancer, including undiagnosed, benign lesion, follicular lesion, follicular neoplasm, possibly malignancy and malignant lesion. However, the difference in the operation technique and experience in the diagnosis leads to missed 
diagnosis, misdiagnosis, and differences in the diagnosis results and pathological examination $(10,11)$.

At present, there are few studies on FNA combined with ultrasound in the diagnosis of thyroid cancer, which was therefore explored in this study, in order to find a more accurate method for the diagnosis of thyroid cancer and provide a more accurate diagnostic reference.

\section{Patients and methods}

Basic information. A retrospective analysis was performed on 165 thyroid nodule patients in Liaocheng People's Hospital (Liaocheng, China) from July 2014 to July 2017, and all patients underwent ultrasound and FNA examinations. The average age was $35.9 \pm 8.7$ years. The patients evaluated in this study were thyroid tumor patients including patients with malignant and benign tumors. There were 147 patients with thyroid malignant tumors, including 119 patients with papillary carcinoma, 14 patients with follicular carcinoma, 8 patients with undifferentiated carcinoma and 6 patients with medullary carcinoma. Another 18 patients had thyroid benign tumors, including 13 patients with nodular goiter, 3 patients with tumor nodules and 2 patients with cysts. Group A consisted of 67 patients with a thyroid nodule diameter of $\leq 1 \mathrm{~cm}$, including 60 patients with malignant nodules and 7 patients with benign nodules, and group B consisted of 98 patients with a thyroid nodule diameter of $>1 \mathrm{~cm}$, including 87 patients with malignant nodules and 11 patients with benign nodules. There were no significant differences in sex, age and BMI between the two groups $(\mathrm{P}>0.05)$ (Table I).

Inclusion and exclusion criteria. Groups A and B included patients diagnosed with malignant or benign thyroid nodules by pathology. These patients were aged 25 to 45 years with stable diseases and vital signs. Patients who underwent ultrasonography and FNA were included. Patients with non-thyroid nodules, patients with unstable diseases, patients who had undergone radiotherapy and chemotherapy, patients complicated with other tumors, patients with severe loss of liver and kidney function, patients with cognitive and communication disorders and patients who did not cooperate with the examination were excluded. All subjects and their family members signed an informed consent form and cooperate with medical staff to complete relevant medical treatment. The study was approved by the Ethics Committee of Liaocheng People's Hospital.

Experimental instruments and materials. Ultrasound diagnosis was performed using the SIEMEAS Acuson sequoia 512 color Doppler ultrasound diagnostic instrument manufactured by GE Healthcare Life Sciences configured with the LA523 high-frequency linear array probe $(12 \mathrm{MHz})$. Lidocaine $(2 \%)$ for puncture and anesthesia was purchased from Changtian Pharma Co., Ltd.; SFDA approval no. H20057825. The biopsy puncture needle $(23 \mathrm{Gx} 8 \mathrm{~cm})$ was purchased from C. R. Bard.

Methods. During the ultrasound examination, the patient was placed in a supine position with the neck raised to fully expose it. The thyroid was scanned thoroughly and versatilely, and the size, location, morphology and calcification of the
Table I. Comparison of general data between two groups of patients $[\mathrm{n}(\%)]$.

\begin{tabular}{|c|c|c|c|c|}
\hline Factors & $\begin{array}{c}\text { Group A } \\
(n=67)\end{array}$ & $\begin{array}{l}\text { Group B } \\
(\mathrm{n}=98)\end{array}$ & $\begin{array}{c}\chi^{2} \\
\text { value }\end{array}$ & $\begin{array}{c}\mathrm{P}- \\
\text { value }\end{array}$ \\
\hline Sex & & & 0.061 & 0.805 \\
\hline Male & $22(32.84)$ & 34 (34.69) & & \\
\hline Female & $45(67.16)$ & $64(65.31)$ & & \\
\hline Age & & & 0.039 & 0.844 \\
\hline$\leq 40$ & $27(40.30)$ & $41(41.84)$ & & \\
\hline$>40$ & $40(59.70)$ & $57(58.16)$ & & \\
\hline BMI $\left(\mathrm{kg} / \mathrm{m}^{2}\right)$ & & & 0.002 & 0.965 \\
\hline$\leq 22$ & $31(46.27)$ & $45(45.92)$ & & \\
\hline$>22$ & $36(53.73)$ & $53(54.08)$ & & \\
\hline Malignant typing & & & 0.651 & 0.885 \\
\hline Papillary carcinoma & $48(71.64)$ & $71(72.45)$ & & \\
\hline Follicular carcinoma & $5(7.46)$ & $9(9.18)$ & & \\
\hline $\begin{array}{l}\text { Undifferentiated } \\
\text { carcinoma }\end{array}$ & $4(5.97)$ & $4(4.08)$ & & \\
\hline Medullary carcinoma & $3(4.48)$ & $3(3.06)$ & & \\
\hline Benign typing & & & 0.144 & 0.931 \\
\hline Nodular goiter & $5(7.46)$ & $8(8.16)$ & & \\
\hline Tumor nodule & $1(1.49)$ & $2(2.04)$ & & \\
\hline Cyst & $1(1.49)$ & $1(1.02)$ & & \\
\hline Family history & & & 0.009 & 0.923 \\
\hline Yes & $49(73.13)$ & $71(72.45)$ & & \\
\hline No & $18(26.87)$ & $27(27.55)$ & & \\
\hline
\end{tabular}

nodule, peripheral and internal blood flow, tissue changes, and changes in surrounding lymph nodes were recorded. After the ultrasound examination, the FNA detection was performed. Before the puncture, the patient was placed in the supine position with head overextension to fully expose the head and neck, then the routine disinfection and drape were performed. Two percent lidocaine was used for local anesthesia and a \#7 needle was used for the negative pressure puncture of the thyroid nodule, in order to obtain the biopsy tissues. After that, the negative pressure was quickly eliminated, the needle was removed and the tissues were sent for examination. The FNA and the ultrasound results were compared with the results of the pathological examination that were used as the gold standard, and the diagnostic values of FNA, ultrasound and FNA combined with ultrasound for thyroid cancer were evaluated. According to relevant studies $(12,13)$, sensitivity, specificity, diagnostic coincidence rate, negative predictive value and positive predictive value were used in this study to evaluate the diagnostic values of FNA, ultrasound and FNA combined with ultrasound for thyroid cancer.

Statistical analysis. The statistical software SPSS 19.0 (IBM Corp., Armonk, NY, USA) was used to analyze and process the research data. $\chi^{2}$ test was used for the comparison of enumeration data between groups. For frequencies $<5$, a Fisher's exact test was used. At $\mathrm{P}<0.05$ the difference was considered statistically significant. 
Table II. Comparison of diagnostic values of ultrasound and ultrasound combined with FNA in group A [n (\%)].

\begin{tabular}{|c|c|c|c|c|}
\hline Diagnosis & Ultrasound & Ultrasound combined with FNA & $\chi^{2}$ value & P-value \\
\hline Sensitivity & $\begin{array}{r}52(86.67) \\
8(13.33)\end{array}$ & $\begin{array}{c}60(100.00) \\
0\end{array}$ & 8.571 & 0.003 \\
\hline Specificity & $\begin{array}{l}2(28.57) \\
5(71.43)\end{array}$ & $\begin{array}{l}2(28.57) \\
5(71.43)\end{array}$ & - & - \\
\hline Accuracy rate & $\begin{array}{l}54(80.60) \\
13(19.40)\end{array}$ & $\begin{array}{c}62(92.54) \\
5(7.46)\end{array}$ & 4.107 & 0.043 \\
\hline Positive predictive value & $\begin{array}{c}52(91.23) \\
5(8.77)\end{array}$ & $\begin{array}{c}60(92.31) \\
6(7.69)\end{array}$ & 0.047 & 0.828 \\
\hline Negative predictive value & $\begin{array}{l}2(25.00) \\
6(75.00)\end{array}$ & $\begin{array}{c}2(100.00) \\
0\end{array}$ & 4.800 & 0.109 \\
\hline
\end{tabular}

FNA, fine-needle aspiration.

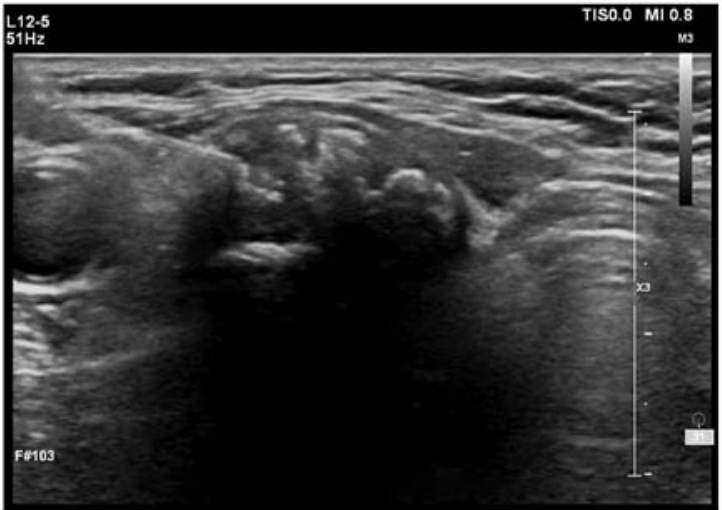

Figure 1. Low echo nodule ultrasound image (papillary carcinoma). Low echo nodule of $1.4 \times 1.3 \times 1.1 \mathrm{~cm}$ was detected by ultrasound, with unclear boundary, heterogeneous internal echoes and multiple dotted high-echoes.

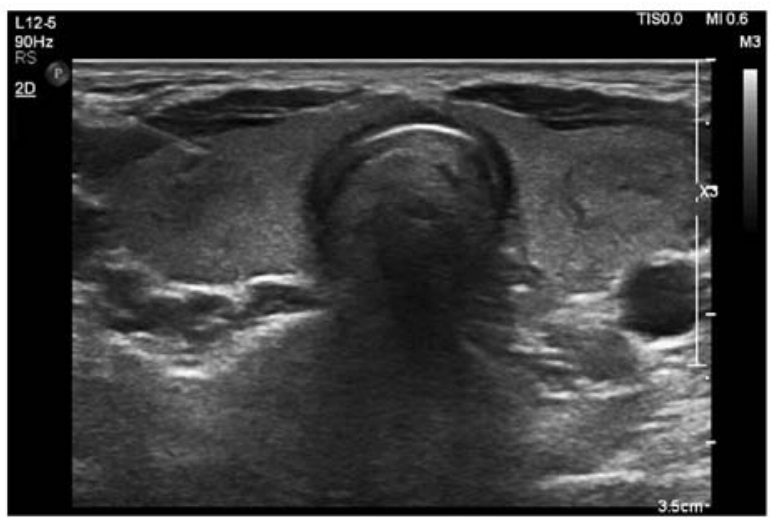

Figure 2. Medium and low echo nodule ultrasound image (papillary carcinoma). Medium and low echo nodules were detected by ultrasound, with an echo halo on the edge and regular shape, therefore suspected of papillary thyroid carcinoma.

\section{Results}

Analysis of partial ultrasoundimagesandFNAimage.Ultrasound images: Fig. 1 shows low echo nodule of $1.4 \times 1.3 \times 1.1 \mathrm{~cm}$ detected

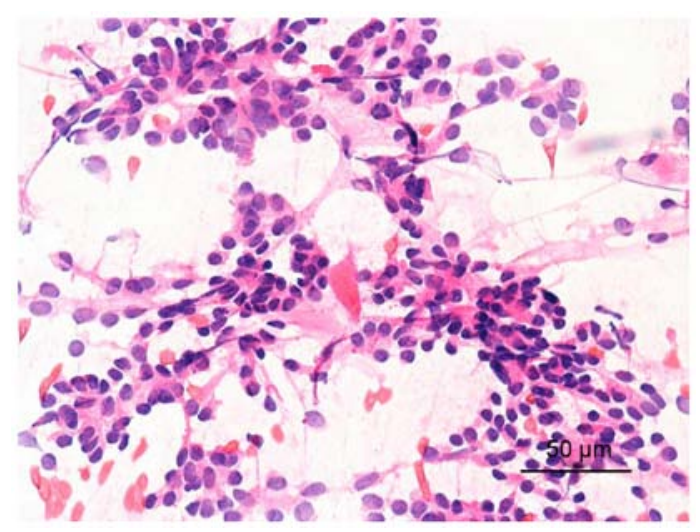

Figure 3. Puncture cytology image (papillary carcinoma) of follicular epithelium. The cells were various in size and crowded, with the nucleus being ground glass-like. Therefore, it was considered as papillary thyroid carcinoma.

by ultrasound, with unclear boundary, heterogeneous internal echoes and multiple dotted high-echoes. Fig. 2 shows medium and low echo nodules detected by ultrasound, with an echo halo on the edge and regular shape, therefore suspected of papillary thyroid carcinoma.

FNA image: Fig. 3 shows follicular epithelium. The cells were various in size and crowded, with the nucleus being ground glass-like. Therefore, it was considered as papillary thyroid carcinoma.

Comparison of diagnostic values of ultrasound, FNA and ultrasound combined with FNA in group A. In group A, the sensitivity, specificity, accuracy, positive predictive value and negative predictive value of ultrasound in the diagnosis of thyroid cancer were $86.67,28.57,80.60,91.23$ and $25 \%$, respectively, those of FNA were 93.33, 71.43, 91.04, 96.55 and $55.56 \%$, respectively, and those of FNA combined with ultrasound were 100, 28.57, 92.54, 95.6 and 100\%, respectively. Ultrasound combined with FNA had higher sensitivity than ultrasound and FNA alone, higher accuracy than ultrasound alone and lower missed diagnosis rate than ultrasound and FNA alone, with statistically significant differences $(\mathrm{P}<0.05)$ (Tables II and III). 
Table III. Comparison of diagnostic values of FNA and ultrasound combined with FNA in group A [n (\%)].

\begin{tabular}{lccr}
\hline Diagnosis & FNA & Ultrasound combined with FNA & $\chi^{2}$ value \\
\hline Sensitivity & $56(93.33)$ & $60(100.00)$ & 4.138 \\
& $4(6.67)$ & 0 & 0.042 \\
Specificity & $5(71.43)$ & $2(28.57)$ & 0.109 \\
& $2(28.57)$ & $5(71.43)$ & 0.099 \\
Accuracy & $61(91.04)$ & $62(92.54)$ & 0.753 \\
Positive predictive value & $6(8.95)$ & $60(7.46)$ & 0.311 \\
& $56(96.55)$ & $5(7.69)$ & 1.029 \\
Negative predictive value & $2(3.45)$ & $2(100.00)$ & $0.31)$ \\
\end{tabular}

FNA, fine-needle aspiration.

Table IV. Comparison of diagnostic values of ultrasound and ultrasound combined with FNA in group B [n (\%)].

\begin{tabular}{lccr}
\hline Diagnosis & Ultrasound & Ultrasound combined with FNA & $\chi^{2}$ value \\
\hline Sensitivity & $84(96.55)$ & $87(100.00)$ & 3.053 \\
& $3(3.45)$ & 0 & 0.081 \\
Specificity & $8(72.73)$ & $7(63.64)$ & 0.210 \\
& $3(27.28)$ & $4(36.37)$ & 0.422 \\
Accuracy & $92(93.88)$ & $94(97.96)$ & 0.516 \\
Positive predictive value & $6(6.12)$ & $2(2.04)$ & 0.106 \\
& $84(96.55)$ & $87(95.61)$ & 0.745 \\
Negative predictive value & $3(3.45)$ & $7(4.39)$ & 2.291 \\
\end{tabular}

FNA, fine-needle aspiration.

Table V. Comparison of diagnostic values of FNA and ultrasound combined with FNA in group B [n (\%)].

\begin{tabular}{lccc}
\hline Diagnosis & FNA & Ultrasound combined with FNA & $\chi^{2}$ value \\
\hline Sensitivity & $87(100.00)$ & $87(100.00)$ & - \\
& 0 & 0 & 0.665 \\
Specificity & $6(54.55)$ & $7(63.64)$ & 0.188 \\
& $5(45.45)$ & $4(36.37)$ & 0.117 \\
Accuracy & $93(94.90)$ & $94(97.96)$ & 0.133 \\
& $5(5.10)$ & $2(2.04)$ & 0.745 \\
Positive predictive value & $87(94.57)$ & $4(4.08)$ & - \\
Negative predictive value & $5(5.43)$ & $7(100.00)$ & - \\
\end{tabular}

FNA, fine-needle aspiration.

Comparison of diagnostic values of ultrasound, FNA and ultrasound combined with FNA in group B. In group $\mathrm{B}$, the sensitivity, specificity, accuracy, positive predictive value and negative predictive value of ultrasound in the diagnosis of 
thyroid cancer were $96.55,72.73,93.88,96.55$ and $72.73 \%$, respectively, those of FNA were 100, 54.55, 94.90, 94.57 and $100 \%$, respectively, and those of FNA combined with ultrasound were 100, 63.64, 97.96, 95.92 and $100 \%$, respectively, without statistically significant differences in those between FNA, ultrasound and FNA combined with ultrasound $(\mathrm{P}>0.05)$ (Tables IV and V).

\section{Discussion}

As the most common thyroid malignant tumor, thyroid cancer accounts for $\sim 1 \%$ of systemic malignant tumors and occurs in patients at any age, with a higher incidence in females than in males (14). In most cases, thyroid cancer occurs on one side of the thyroid gland and is a single tumor (11). In clinical practice, the early clinical manifestations of thyroid cancer are similar to those of benign thyroid nodules, which are mainly lumps in the thyroid, difficult breathing, swallowing obstruction and hoarseness, so thyroid cancer is easily misdiagnosed as a benign nodule. Therefore, the malignant lesion should be detected and excluded for each thyroid nodule patient $(15,16)$. The most common pathological type of thyroid cancer is papillary adenocarcinoma that accounts for $\sim 70 \%$, followed by follicular adenocarcinoma, medullary carcinoma and undifferentiated carcinoma (17). At present, the key to the treatment of thyroid cancer remains how to make the early diagnosis and intervention therapy (18). Ultrasound, which is commonly used in clinical practice, is an auxiliary diagnostic method of thyroid cancer, but the ultrasound images of thyroid cancer are overlapping and diverse, so ultrasound has a high misdiagnosis rate of atypical and early thyroid cancers (19). Simple to operate and relatively safe, FNA has higher sensitivity and accuracy and is currently considered to be effective for detecting thyroid cancer worldwide (20). However, the diagnosis of some nodules by FNA such as follicular adenocarcinomas is unclear (21). Therefore, in this study, the diagnostic value of FNA combined with ultrasound for thyroid cancer was explored, in order to provide a more accurate diagnostic method of thyroid cancer.

In this study, the diagnostic values of ultrasound, FNA and ultrasound combined with FNA for patients with a thyroid nodule $\leq 1 \mathrm{~cm}$ were first analyzed. The results showed that ultrasound combined with FNA had higher sensitivity than ultrasound and FNA alone, higher accuracy than ultrasound alone $(\mathrm{P}<0.05)$. There is a study (22) showing that preoperative ultrasound combined with ultrasound-guided lymph node puncture biopsy can detect approximately $>50 \%$ of breast cancer patients with axillary lymph node metastasis, which indicates the diagnostic value of ultrasound combined with puncture biopsy, and supports our conclusion. Then, the diagnostic values of ultrasound, FNA and ultrasound combined with FNA for patients with a thyroid nodule $>1 \mathrm{~cm}$ were analyzed. The results showed that in group B $(>1 \mathrm{~cm}$ group), there were no statistically significant differences in the sensitivity, specificity, accuracy, positive predictive value and negative predictive value between FNA, ultrasound and FNA combined with ultrasound $(\mathrm{P}>0.05)$. These findings show that for large nodules, ultrasound, FNA and ultrasound combined with FNA are not much different, but ultrasound is relatively more convenient and economical. There is a study (23) showing that the accuracy of FNA is $100.0 \%$ in the diagnosis of medullary thyroid carcinoma with a diameter $>1 \mathrm{~cm}$ and $66.6 \%$ in the diagnosis of medullary microcarcinoma with a diameter $<1 \mathrm{~cm}$, which is similar to our findings. We concluded that the accuracy of ultrasound and FNA is affected by the size of the nodule, and the smaller the nodule is, the lower the accuracy of diagnosis is, which are also consistent with the findings of Shrestha et al (24). However, it has been reported (25) that the sensitivity of thyroid FNA affected by operational techniques and diagnostic experience is difficult to be calculated accurately. Therefore, our experimental results have yet to be further verified.

In summary, FNA combined with ultrasound can significantly improve the sensitivity and accuracy in the diagnosis of the thyroid nodule in the $\leq 1 \mathrm{~cm}$ group, while ultrasound, FNA and ultrasound combined with FNA all have higher sensitivity and accuracy in the diagnosis of the thyroid nodule in the $>1 \mathrm{~cm}$ group, without significant differences. Therefore, it can be considered that ultrasound combined with FNA has a higher diagnostic value for the determination of most benign and malignant thyroid nodules, and can be used as a preferred solution for the clinical diagnosis of thyroid cancer.

\section{Acknowledgements}

Not applicable.

\section{Funding}

No funding was received.

\section{Availability of data and materials}

The datasets used and/or analyzed during the present study are available from the corresponding author on reasonable request.

\section{Authors' contributions}

JL wrote the manuscript. JL and QW collected and interpreted the data. JL, QW and LW were mainly devoted to surgery. JW and DW analyzed the FNA results. ZX, YL and QZ were responsible for and the ultrasound results. All the authors read and approved the final manuscript.

\section{Ethics approval and consent to participate}

The study was approved by the Ethics Committee of Liaocheng People's Hospital (Liaocheng, China). The signed informed consents were obtained from the patients or the guardians.

\section{Patient consent for publication}

Not applicable.

\section{Competing interests}

The authors declare that they have no competing interests. 


\section{References}

1. Giordano D, Valcavi R, Thompson GB, Pedroni C, Renna L, Gradoni $\mathrm{P}$ and Barbieri V: Complications of central neck dissection in patients with papillary thyroid carcinoma: Results of a study on 1087 patients and review of the literature. Thyroid 22: 911-917, 2012.

2. Lee HS, Park C, Kim SW, Song JW, Chun BK, Park TJ, Hong JC and Lee KD: Primary tumour characteristics predict the invasiveness of lymph node metastases in papillary thyroid carcinoma patients. J Laryngol Otol 130: 302-308, 2016.

3. James BC, Mitchell JM, Jeon HD, Vasilottos N, Grogan RH and Aschebrook-Kilfoy B: An update in international trends in incidence rates of thyroid cancer, 1973-2007. Cancer Causes Control 29: 465-473, 2018.

4. Tang W, Huang C, Tang C, Xu J and Wang H: Galectin-3 may serve as a potential marker for diagnosis and prognosis in papillary thyroid carcinoma: A meta-analysis. OncoTargets Ther 9: 455-460, 2016.

5. Archier A, Heimburger C, Guerin C, Morange I, Palazzo FF, Henry JF, Schneegans O, Mundler O, Abdullah AE, Sebag F, et al: (18)F-DOPA PET/CT in the diagnosis and localization of persistent medullary thyroid carcinoma. Eur J Nucl Med Mol Imaging 43: 1027-1033, 2016.

6. Li F, Zhang J, Wang Y and Liu L: Clinical value of elasticity imaging and contrast-enhanced ultrasound in the diagnosis of papillary thyroid microcarcinoma. Oncol Lett 10: 1371-1377, 2015.

7. Trimboli P, Nasrollah N, Amendola S, Rossi F, Ramacciato G, Romanelli F, Aurello P, Crescenzi A, Laurenti O, Condorelli E et al: Should we use ultrasound features associated with papillary thyroid cancer in diagnosing medullary thyroid cancer? Endocr J 59: 503-508, 2012.

8. Niu LJ, Hao YZ and Zhou CW: Diagnostic value of ultrasonography in thyroid lesions. Zhonghua Er Bi Yan Hou Tou Jing Wai Ke Za Zhi 41: 415-418, 2006 (In Chinese).

9. Hong YR, Luo ZY, Mo GQ, Wang P, Ye Q and Huang PT: Role of contrast-enhanced ultrasound in the pre-operative diagnosis of cervical lymph node metastasis in patients with papillary thyroid carcinoma. Ultrasound Med Biol 43: 2567-2575, 2017.

10. Theoharis C, Adeniran AJ, Roman S, Sosa JA and Chhieng D: The impact of implementing The Bethesda System for reporting of thyroid FNA at an academic center. Diagn Cytopathol 41 : 858-863, 2013.

11. Renshaw AA and Gould EW: Why there is the tendency to 'overdiagnose' the follicular variant of papillary thyroid carcinoma. Am J Clin Pathol 117: 19-21, 2002.

12. Ruilong Z, Daohai X, Li G, Xiaohong W, Chunjie W and Lei T: Diagnostic value of 18F-FDG-PET/CT for the evaluation of solitary pulmonary nodules: A systematic review and meta-analysis. Nucl Med Commun 38: 67-75, 2017.
13. Liu X, Ouyang D, Li H, Zhang R, Lv Y, Yang A and Xie C: Papillary thyroid cancer: Dual-energy spectral CT quantitative parameters for preoperative diagnosis of metastasis to the cervical lymph nodes. Radiology 275: 167-176, 2015.

14. Sipos JA: Advances in ultrasound for the diagnosis and management of thyroid cancer. Thyroid 19: 1363-1372, 2009.

15. Levy I, Barki Y and Tovi F: Giant cervical cyst: Presenting symptom of an occult thyroid carcinoma. J Laryngol Otol 105: 863-864, 1991.

16. Vierhapper H, Niederle B, Bieglmayer C, Kaserer K and Baumgartner-Parzer S: Early diagnosis and curative therapy of medullary thyroid carcinoma by routine measurement of serum calcitonin in patients with thyroid disorders. Thyroid 15: 1267-1272, 2005

17. Sheils O: Molecular classification and biomarker discovery in papillary thyroid carcinoma. Expert Rev Mol Diagn 5: 927-946, 2005.

18. Huang GQ, Liu Y, Cao DF, Gong Y, Su D, Zhao JH and Wang L: Advances in tumor markers for the early diagnosis of papillary thyroid carcinoma. Int J Pharm Pharm Sci 8: 47, 2016.

19. Harshan M, Crapanzano JP, Aslan DL, Vazquez MF and Saqi A: Papillary thyroid carcinoma with atypical histiocytoid cells on fine-needle aspiration. Diagn Cytopathol 37: 244-250, 2009.

20. Trimboli P, Treglia G, Guidobaldi L, Romanelli F, Nigri G, Valabrega S, Sadeghi R, Crescenzi A, Faquin WC, Bongiovanni $\mathrm{M}$, et al: Detection rate of FNA cytology in medullary thyroid carcinoma: A meta-analysis. Clin Endocrinol (Oxf) 82: 280-285, 2015.

21. Yoon JH, Kim EK, Kwak JY and Moon HJ: Effectiveness and limitations of core needle biopsy in the diagnosis of thyroid nodules: Review of current literature. J Pathol Transl Med 49: 230-235, 2015

22. Houssami N, Ciatto S, Turner RM, Cody HS III and Macaskill P: Preoperative ultrasound-guided needle biopsy of axillary nodes in invasive breast cancer: Meta-analysis of its accuracy and utility in staging the axilla. Ann Surg 254: 243-251, 2011.

23. Yang GC, Fried K and Levine PH: Detection of medullary thyroid microcarcinoma using ultrasound-guided fine needle aspiration cytology. Cytopathology 24: 92-98, 2013.

24. Shrestha M, Crothers BA and Burch HB: The impact of thyroid nodule size on the risk of malignancy and accuracy of fine-needle aspiration: A 10-year study from a single institution. Thyroid 22: 1251-1256, 2012.

25. Pitman MB, Abele J, Ali SZ, Duick D, Elsheikh TM, Jeffrey RB, Powers CN, Randolph G, Renshaw A and Scoutt L: Techniques for thyroid FNA: a synopsis of the National Cancer Institute Thyroid Fine-Needle Aspiration State of the Science Conference. Diagn Cytopathol 36: 407-424, 2008.

(C) (i) () This work is licensed under a Creative Commons

EY NO NO Attribution-NonCommercial-NoDerivatives 4.0 International (CC BY-NC-ND 4.0) License. 